\title{
Heterostructure Silicon and Germanium Alloy Based Thin Film Solar Cell Efficiency Analysis
}

\author{
Ashish Kumar Singh ${ }^{*}$, Manish Kumar, Dilip Kumar, S N Singh \\ Department of Electronics \& Communication Engineering National Institute of Technology, Jamshedpur and \\ 831014, India
}

Received: 01 December 2019; Accepted: 27 January 2020; Published: 08 April 2020

\begin{abstract}
Thin film solar cell along with enhanced absorption property will be the best, so combination of SiGe alloy is considered. The paper presented here consists of a numerical model of $\mathrm{Si} / \mathrm{Si}_{1-\mathrm{x}} \mathrm{Ge}_{\mathrm{x}}$ heterojunction solar cell. The addition of Ge content to Si layer will affect the property of material. The research has investigated characteristics such as short circuit current density (JSC), generation rate $G$, absorption coefficient $(\alpha)$, and open circuit voltage $\left(V_{o c}\right)$, power, fill factor $(F F)$ with optimal Ge concentration. The speculative determination of appropriate germanium mole fraction is done to get the maximized thin-film solar cell efficiency.
\end{abstract}

Index Terms: vegard's law, short-circuit current density, Saturation current (Is), Generation Rate, Absorption Coefficient and open circuit voltage.

(C) 2020 Published by MECS Publisher. Selection and/or peer review under responsibility of the Research Association of Mode rn Education and Computer Science

* Corresponding author.

E-mail address: ashishsingh0530@gmail.com 


\section{Introduction}

The renewable source of energy generated from solar cell plays a vital role in near future. Crystalline silicon semiconductor is less preferable for solar cell because of its indirect band structure resulting the low absorption coefficient. Therefore, silicon heterojunction junction is preferred with large band gap for high performance by achieving high electrical voltage [1]. Solar cell material is decided on the basis of the good spectrum absorption. The optical absorption coefficient of Ge is higher than that of Si and the energy band gap of Si is higher than Ge. This makes the alloy to have trade-off between absorption coefficient and energy band gap [2]. The increment of Ge content in the alloy result in increment in absorption at longer wavelength and decrement in resulting band gap. This method is used for speculating the exact germanium mole fraction for achieving highest efficiency before fabrication. Here we have considered trapping at the interfaces while simulating the performance. In previous model [3] result has been published without considering trapping. The limitation of previous research was the efficiency curve, without trapping the curve will not show the exact behaviour as compared to the real scenario. This model will provide the approximately exact efficiency characteristics that can be useful in future research for boosting efficiency. In Section 2, the system model has been discussed. Simulation results are presented under Section 3 with simulated data and efficiency comparison.

\section{System Model}

The air mass coefficients (AM) have been defined for comparing the solar module performance under standard test conditions. Figure 1 show the spectral irradiance curve with top most curve representing solar spectrum outside the earth's atmosphere also known as air mass zero condition (AM0) which reaches the earth's surface directly. The middle and bottom curve represent AM1.5 Global and AM1.5 Direct, respectively.

The model investigated is of form $\mathrm{Si} / \mathrm{Si}_{1-\mathrm{x}} \mathrm{Ge}_{\mathrm{x}}$ where $\mathrm{x}$ represents the mole fraction of germanium. The n-on-p model energy band diagram is shown in figure 2. The valance offset $\Delta E_{v}$ plays important role while calculation of emission rate. The thin film Heterostructure solar cell [3] is show in Fig. 3, where most of the absorption took place at SiGe layer. At top most layer antireflection coating of indium tin oxide is used to allow maximum light through it. A highly doped thin layer of $5 \mathrm{~nm} n$ - type silicon act as luminescence efficiency booster [4]. Thickness of SiGe layer is assigned different values while the bottom p type silicon substrate is of $100 \mathrm{~nm}$. A photon having energy less than the band gap do not contribute whereas photon with energy greater than or equal to band gap $\left(E_{g}\right)$ generates electron hole pairs within the active region. These generated electrons experiences diffusion (concentration difference) and drift (applied voltage) to generate photocurrent. Table 1 summaries important parameters used for simulation. Here we will consider the case of holes first using coordinate assumption as given in [3].

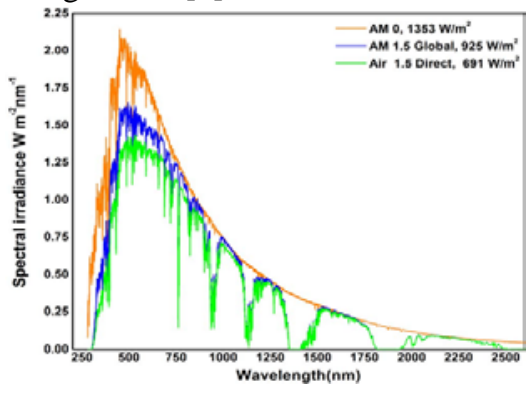

Fig.1. Solar spectral irradiance 


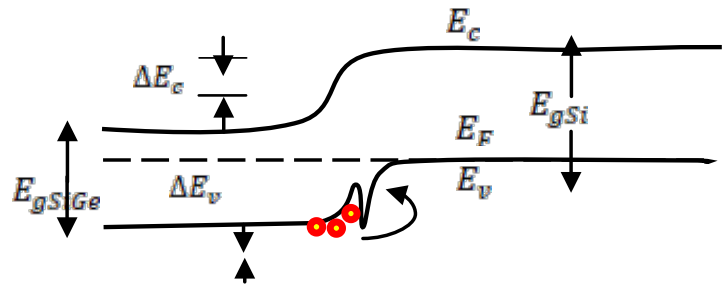

Fig.2. Energy band diagram

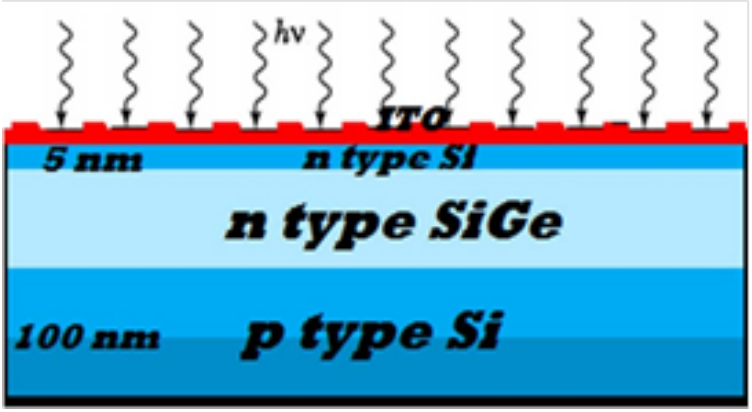

Fig 3. Si/SiGe Heterostructure

Table 1. Parametric value used in simulation

\begin{tabular}{lc}
\hline \multicolumn{1}{c}{ Parameters } & Value \\
\hline Concentration $(\mathrm{SiGe})$ & $3 \times 10^{25} \mathrm{~m}^{-3}$ \\
Concentration $(\mathrm{Si})$ & $3 \times 10^{22} \mathrm{~m}^{-3}$ \\
Device Area $(\mathrm{A})$ & $4 \times 10^{-4} \mathrm{~m}^{2}$ \\
Mobility Si $\left(\mu_{\mathrm{n}}\right)$ & $270 \times 10^{-4}$ \\
Mobility Si $\left(\mu_{\mathrm{p}}\right)$ & $95 \times 10^{-4}$ \\
Mobility Ge $\left(\mu_{\mathrm{n}}\right)$ & $350 \times 10^{-4}$ \\
Mobility Ge $\left(\mu_{\mathrm{p}}\right)$ & $80 \times 10^{-4}$ \\
$\mathrm{Na}_{\mathrm{N}}$ & $1.5 \times 10^{22} \mathrm{~m}^{-3}$ \\
$\mathrm{~N}_{\mathrm{d}}$ & $5 \times 10^{25} \mathrm{~m}^{-3}$ \\
$\tau_{\mathrm{p}}$ & $1 \times 10^{-8} \mathrm{~s}$ \\
$\mathrm{~d}_{\mathrm{Si}}$ & $100 \times 10^{-9} \mathrm{~m}$ \\
\end{tabular}

The following equation (1-15) is used for generating a numerical model in MATLAB. Using the differential equations, we have calculated the exact solution for diffusion current density and used it further for computation of efficiency. Combining one-dimensional steady-state continuity equation for hole in n-side under low injection condition with current density equation (assuming no electric field) is

$$
G_{p}-\frac{p_{n}-p_{n g}}{\tau_{p}}-\frac{1}{q} \frac{d}{d x}\left(-q D_{p} \frac{d p_{n}}{d x}\right)=0
$$

Where $G_{P}$ is the generation rate of holes with $p_{n o}$ density of hole at equilibrium and $p_{n}$ density under 
illumination. $D_{p}$ is the diffusion coefficient of hole for n-SiGe

$$
G(\lambda, X)=\alpha(\lambda) F(\lambda)[1-R(\lambda)] e^{-\alpha(\lambda) x}
$$

Substituting value of G in Eq.1 from Eq. 2 we get differential equation as follows

$$
\frac{d^{2} p_{n}}{d x^{2}}-\frac{p_{n}}{\tau_{p} D_{p}}-\frac{p_{n 0}}{\tau_{p} D_{p}}+\frac{\alpha(\lambda) F(\lambda)[1-R(\lambda)] e^{-\alpha(\lambda) x}}{D_{p}}
$$

The solution of differential Eq.3 is of form

$y(x)=\varkappa_{1} e^{m x}+\varkappa_{2} e^{-m x}$

where $\mathrm{m}$ is the homogenous root of different equation. Let us assume the value of constants

$x_{1}=\frac{\omega+\psi}{2}$ and $x_{2}=\frac{\omega-\psi}{2}$

Substituting value of constants in Eq.4 from Eq. 5 and after few manipulations we get equation

$$
y(x)=\omega \cosh (m x)+\psi \sinh (m x)
$$

Therefore using Eq. (4-6) the general solution of Eq.3 is given as

$$
p_{n}-p_{n o}=\omega \cosh \left(\frac{x}{\sqrt{\tau_{p} D_{p}}}\right)+\psi \sinh \left(\frac{x}{\sqrt{\tau_{p} D_{p}}}\right)-\frac{\alpha(\lambda) F(\lambda)[1-R(\lambda)]^{-m(x) x}}{\alpha^{2} \tau_{p} D_{p}-1}
$$

where $L_{p}=\sqrt{\tau_{p} D_{p}}$ is the diffusion length of holes in n-SiGe. Now applying boundary condition at surface [4] we get final diffusion current density for holes at depletion edge as a function of wavelength

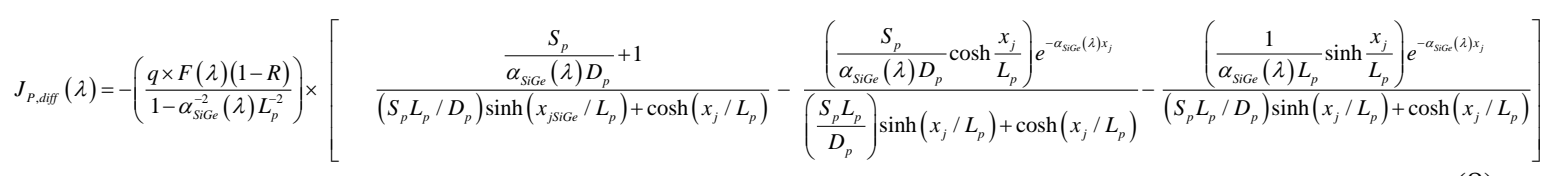

where reflectivity $R$ is the fraction of photons reflected from surface, $F(\lambda)$ is the number of incident photons per area per second, $\alpha_{\text {SiGe }}(\lambda)$ is the absorption coefficient of SiGe layer and $S_{p}$ is the surface recombination velocity. Similarly to find electron photocurrent at depletion edge from the base of the cell we again apply boundary condition [4] and get

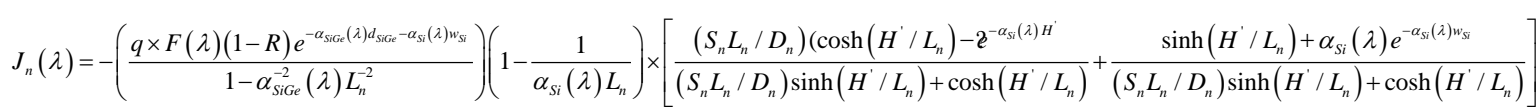


where $H^{\prime}$ is the length of $\mathrm{p}$ type $\mathrm{Si}$ region, $D_{n}$ is the diffusion coefficient of electron for $\mathrm{p}-\mathrm{Si}, \alpha_{S i}(\lambda), L_{n}$ and $S_{n}$ are the absorption coefficient of silicon, diffusion length of electron in $\mathrm{p}$ type and recombination velocity respectively. Within depletion region the electric field is high which accelerate out the photo generated current inside the region before their recombine. The resultant drift current density as a function of wavelength is given as

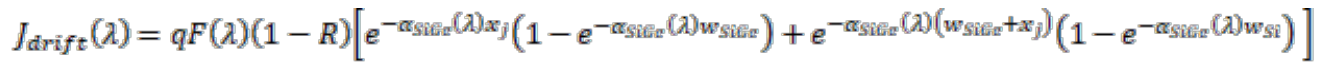

where $w_{S i G e}$ and $w_{S i}$ are depth of depletion region exceeded into n-SiGe region and p-Si region respectively. The heterostructure cause valance band discontinuity $\Delta E_{v}$ as shown in Fig.2, therefore holes get trapped at the n-SiGe/pSi interface. The holes from this trap are emitted by a slow thermionic emission process and, thus, take a long time to reach the contacts [1]. The trapping for long time results in recombination and hence carrier loss occurs resulting a decrease in the photocurrent. Therefore efficiency is also decreased as a result of downfall of overall photocurrent. Thermionic emissions process is dominant in solar cell as it get operated at high temperature and it is govern by

$$
\varepsilon_{e p}=\xi e^{\frac{-\Delta \mathbb{R}_{v}}{k t}}
$$

where $\varepsilon_{e p}$ is the holes emission rate, $\mathrm{k}$ is the Boltzman constant, $\mathrm{T}$ is the operational temperature in $\mathrm{K}$, and $\xi$ is a constant. For the speculation of holes emission rate we require $\Delta E_{v}$ through:

$$
\Delta E_{g}=\Delta E_{v}+\Delta E_{c}
$$

The valence band offsets are calculated assuming very small constant value of $\Delta E_{c}, 0.02 \mathrm{eV}[1]$ and using experimental data band gap difference is calculated [5]. The factor $\delta_{h}$ by which the holes photocurrent decreases is

$$
\delta_{h}=\frac{\varepsilon_{\varepsilon p}}{\varepsilon_{\varepsilon p}+\varepsilon_{r p}}
$$

Where $\varepsilon_{r p}$ is the band-to-band recombination rate of holes. Here we have only considered the effect of trapping in diffusion component. The total current density $I(\lambda)$ can be written as the summation of diffusion and drift density:

$$
I(\lambda)=\delta_{h} \times J_{p}(\lambda)+J_{d r}(\lambda)+J_{n}(\lambda)
$$

By integrating $J(\lambda)$ over full range of solar radiation we obtain total photocurrent and total current can be defined as

$$
I=J_{0}\left(e^{q v / k T}-1\right)-\int_{\lambda_{1}}^{\lambda_{2}} J(\lambda) d \lambda
$$

Where, $I_{0}$ is the saturation current density.

\section{Simulation Results}


The numerical model is simulated using parameters used in [1,6,7] and analysis result has been produced under this section. For simulation purpose we have collected the different properties of silicon, germanium, the number of incident photons per area per second and used vegard law for getting the properties of combination. The absorption curve is the resultant of vegard law applied to alpha $n$ silicon and alpha $n$ germanium. The optical properties of silicon is important to analyse the overall properties of heterostructure solar cell.

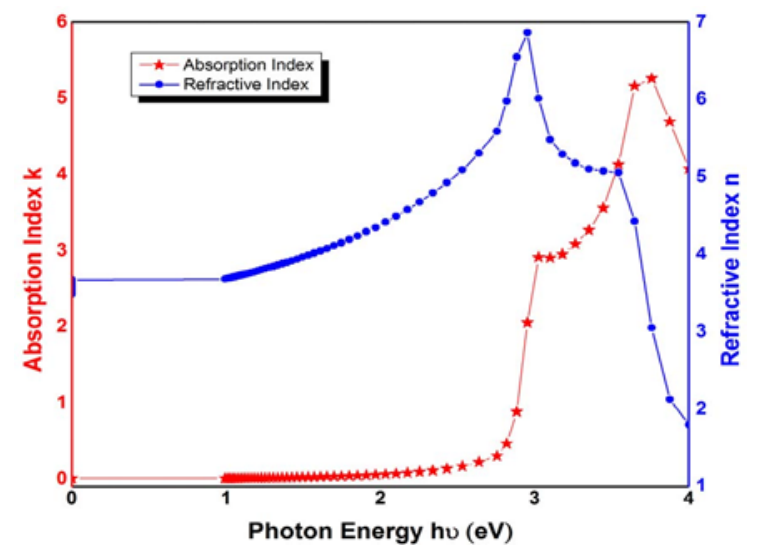

Fig.4. Characteristic of Silicon

When a particular wavelength of light propagates through given refractive index material, the measure of attenuation caused by absorption of energy per unit of distance is termed as absorption index. Absorption index and refractive index of silicon is shown in Fig.4. Both are derived from same physical process in a material. For the same wavelength of light material with large band gap will have smaller refractive index i.e. light move faster. Therefore we required to increase index of refraction by decreasing overall band gap along with increasing absorption index. The term absorption coefficient or extinction coefficient is intimately related which is important in deciding the solar cell material. It gives us an idea of how far the incident light with energy higher than threshold penetrates inside the material before getting absorbed. If we compare germanium and silicon over absorption coefficient former one has wider spectral overlap with solar spectrum. Adding Ge content enhances response over longer wavelength by improving absorption coefficient of overall device. This implies generation of large number of electron hole pairs and results high short circuit current density to enhance the efficiency.

Fig. 5 depicts the absorption coefficient of alloy at different mole fraction of Ge for the given photon energy. This figure also depict the absorption coefficient of $\mathrm{Si}$, Ge when mole fraction of Ge is lowest and fraction is highest simultaneously. The majority of photons is absorbed at the surface of the solar cell material therefore the generation rate is maximum at surface. The absorption of photos result in generation of electrons in the cell and that number of generated electron at each point of device is termed as generation rate. Fig. 6 present the generation rate of the alloy at different Ge content. For the computation of current density as a function of voltage we theoretically changed the thickness of the alloy layer. In fig. 7 the plotted curve shows the variation in current density at different Ge content for the given device alloy thickness of $25 \mathrm{~nm}$. As a result of simulation of Eq. 15 we have to find maximum area under $I_{s c}$ vs. V curve. Fig. 7 depicts that the larger mole fraction of $\mathrm{Ge}$, lesser is the current density. But due to the absorption and carrier trapping combined effect, the Ge mole fraction "0.15" current density becomes maximum i.e. $264.76 \mathrm{~A} / \mathrm{m} 2$ resulting in open circuit voltage of $0.551 \mathrm{~V}$. 


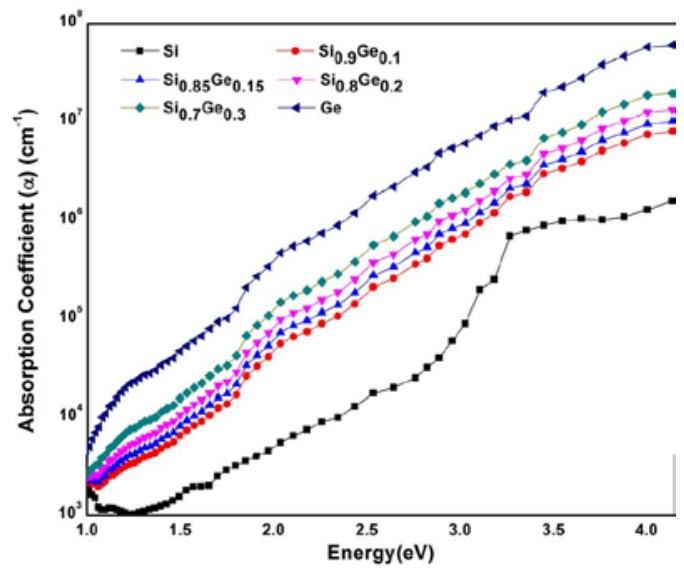

Fig.5. SiGe absorption coefficient as a function of photon energy



Fig.6. Generation rate as a function of surface distance

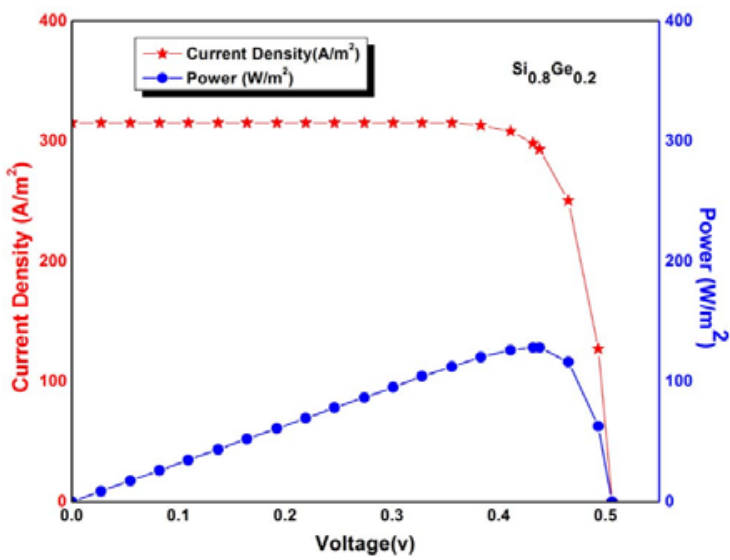

Fig.7. Current density as a function of voltage for $\mathrm{dSiGe}=25 \mathrm{~nm}$ 




Fig.8. Current density as a function of voltage for $\mathrm{dSiGe}=50 \mathrm{~nm}$

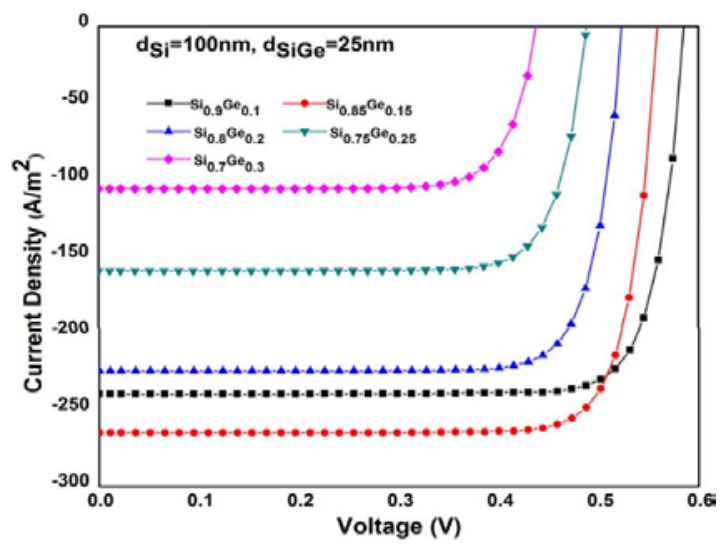

Fig.9. Current density and power vs. Voltage curve

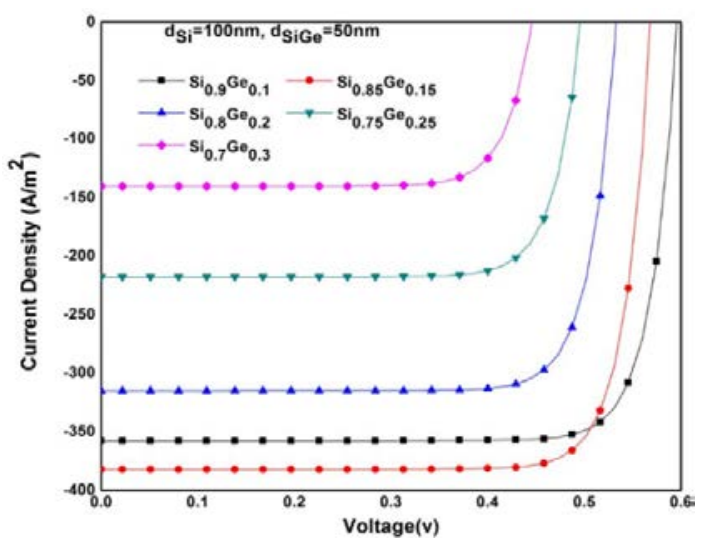

Fig.10. Ge content-based efficiency curve for different alloy thickness 
Whereas for $50 \mathrm{~nm}$ thickness of alloy the current density for different Ge content is observed to be increased. From fig. 8 we can conclude that current density of device is increasing with decrease in Ge mole fraction. But again due to trapping and absorption phenomenon the curve behaves exceptionally for the $\mathrm{Ge}$ mole fraction" 0.15 ” and produce maximum current density of $382.50 \mathrm{~A} / \mathrm{m} 2$ resulting in open circuit voltage of $0.56 \mathrm{~V}$. The important cell electrical parameters which contributes in efficiency of solar cell are short circuit current density $\left(J_{\sigma r}\right)$, open circuit $\left(V_{o c}\right)$, maximum output power $\left(P_{m}\right)$ and fill factor $(F F)[8,9,10]$. The maximum output power can be calculated through fig. 9 by getting value of maximum current $\left(I_{m v}\right)$ and maximum voltage $\left(V_{m v}\right)$.

Table 2 data has been taken after simulation of Eq. (1-15). MATLAB has been used for the numerical model simulation of proposed heterojunction solar cell. After computation of current density, open circuit voltage and fill factor for different mole fraction of germanium we can conclude that for getting maximum efficiency germanium mole fraction of " 0.1 " is the most suitable combination.

Table 2. Parameters values obtained after simulation for different Ge mole fraction

\begin{tabular}{|c|c|c|c|c|c|c|c|c|}
\hline \multirow{2}{*}{$\begin{array}{c}\text { Ge mole } \\
\text { Fraction(x) } \\
\text { Si1-xGex }\end{array}$} & \multicolumn{4}{|c|}{ dSiGe $=25 \mathrm{~nm}, \quad d S i=100 \mathrm{~nm}$} & \multicolumn{4}{|c|}{$\mathrm{dSiGe}=50 \mathrm{~nm}, \mathrm{dSi}=100 \mathrm{~nm}$} \\
\hline & $\begin{array}{c}J_{S C} \\
\left(A / m^{2}\right)\end{array}$ & $\begin{array}{l}\text { Voc } \\
(V)\end{array}$ & $\begin{array}{c}\text { Fill } \\
\text { Factor }\end{array}$ & $\begin{array}{c}\text { Efficiency } \\
\text { (\%) }\end{array}$ & $\begin{array}{c}J_{S C} \\
\left(A / m^{2}\right)\end{array}$ & $\begin{array}{l}\text { Voc } \\
(V)\end{array}$ & $\begin{array}{c}\text { Fill } \\
\text { actor }\end{array}$ & $\begin{array}{c}\text { Efficiency } \\
\text { (\%) }\end{array}$ \\
\hline 0.1 & 239.43 & 0.589 & 0.825 & 11.63 & 358.18 & 0.590 & 0.839 & 17.73 \\
\hline 0.15 & 264.76 & 0.551 & 0.816 & 11.9 & 382.50 & 0.560 & 0.816 & 17.47 \\
\hline 0.2 & 224.58 & 0.509 & 0.805 & 9.2 & 315.32 & 0.521 & 0.804 & 13.2 \\
\hline 0.25 & 159.29 & 0.488 & 0.8 & 6.22 & 218.06 & 0.490 & 0.8 & 8.54 \\
\hline 0.3 & 105.63 & 0.439 & 0.784 & 3.64 & 140.57 & 0.458 & 0.79 & 5.08 \\
\hline
\end{tabular}

The fill factor is defined as

$$
F F=\frac{I_{m} V_{m}}{I_{s c} V_{o c}}
$$

Therefore, under AM 1.5 the conversion efficiency of the device is give as

$$
\eta=\frac{I_{m} V_{m}}{P_{i n}}
$$

Using Eq. 16 and Eq. 17 we get the final expression for efficiency as

$$
\eta=\frac{I_{s c} V_{m} F F}{P_{i n}}
$$

To maximize the efficiency of the heterostructure solar cell we have to maximize all three terms in the numerator of Eq. 18[11]. Using parameters from table 3 in Eq. 18, efficiency curve at different alloy thickness can be plotted by fixing the thickness of Si to $100 \mathrm{~nm}$. Fig. 10 shows the variation of efficiency of solar cell at different Ge mole fraction. Increase in alloy thickness resulted in boosting of cell efficiency. At lower value of Ge mole fraction the effect of absorption is dominating but as the fraction increases, carrier trappingsuppress the dominating effect of absorption. 


\section{Conclusion}

A detail study has been done on the $\mathrm{Si} / \mathrm{Si}_{1-\mathrm{x}} \mathrm{Ge}_{\mathrm{x}}$ heterostructure solar cell to investigate the efficiency variation at different $\mathrm{Ge}$ mole fraction. The active layer responsible for the absorption was $\mathrm{Si}_{1-\mathrm{x}} \mathrm{Ge}_{\mathrm{x}}$ alloy layer. From fig. 10 it can be concluded that at $50 \mathrm{~nm}$ alloy layer thickness, the solar cell efficiency attains greater value than the efficiency at $25 \mathrm{~nm}$ of alloy thickness. In both the case at certain mole fraction, increasing efficiency curve attains maxima and then it start decreasing. The reason behind the bending of efficiency curve is due to absorption as well as carrier trapping. As the germanium mole fraction is increased the absorption keeps on increasing causing more number of holes trapping in valence offset band. At high absorption, trapping effect comes into play and will result in downfall of overall photocurrent due to recombination. Therefore we can say that efficiency is a function of Ge mole fraction in the $\mathrm{Si}_{1-\mathrm{x}} \mathrm{Ge}_{\mathrm{x}}$ alloy layer which depends on the thickness of top $\mathrm{Si}$ layer and $\mathrm{Si}_{1-\mathrm{x}} \mathrm{Ge}_{\mathrm{x}}$ layer. The maximum efficiency is found approximately between $x=0.12$ and 0.15 . Overall this method is useful for getting results when variation in material layer, temperature, device configuration and composition of thin film solar has to be studied.

\section{Acknowledgements}

The authors wish to acknowledge Dr. Mukul Kumar Das, Associate Professor in Department of Electronics Engineering, Indian Institute of Technology (IIT-ISM) Dhanbad for the guidance.

\section{References}

[1] M. K. Das and S. K. Choudhary, "Ge-content dependent efficiency of Si/SiGe heterojunction solar cell," in Proceeding of the Photonics Global Conference (PGC '12), pp. 1-4, Singapore, December 2012.

[2] Md Sadullah, Jaspinder Kaur, Rikmantra Basu, Ajay K. Sharma, Analysis of thin-film direct band-gap SiGeSn alloy based heterostructure solar cell featuring SiGe absorber layer,Optik,Volume 202,2020,163715,ISSN 0030-4026,https://doi.org/10.1016/j.ijleo.2019.163715.

[3] Ashish Kumar Singh, Jahnvi Tiwari, Ashish Yadav, and Rakesh Kumar Jha, “Analysis of Si/SiGe Heterostructure Solar Cell,” Journal of Energy, vol. 2014, Article ID 946406, 7 pages, 2014. doi:10.1155/2014/946406

[4] S.M. Sze. Physics of Semiconductor Devices. J. Wiley, New York, 2nd edition, 1982.

[5] R. People, Physics and application of Gex Si1-x /Si strained-layer heterostructures. IEEE J. Quantum Electron. 22, 1696 (1986)

[6] Ming Han Liao; Chen, C.H., "The Investigation of Optimal Si-SiGe Hetero-Structure Thin-Film Solar Cell With Theoretical Calculation and Quantitative Analysis," Nanotechnology, IEEE Transactions on , vol.10, no.4, pp.770,773, July 2011 doi: 10.1109/TNANO.2010.2077647

[7] Ashish Kumar Singh, Jahnvi Tiwari, Ashish Yadav, and Rakesh Kumar Jha, "MATLAB User Interface for Simulation of Silicon Germanium Solar Cell,” Journal of Materials, vol. 2015, Article ID 840718, 6 pages, 2015. 
[8] Somenath Chatterjee, Sumeet Singh, and Himangshu Pal, "Effect of Multijunction Approach on Electrical Measurements of Silicon and Germanium Alloy Based Thin-Film Solar Cell Using AMPS-1D,” International Journal of Photoenergy, vol. 2014, Article ID 653206, 6 pages, 2014. doi:10.1155/2014/653206.

[9] S. S.Hegedus, "Current—voltage analysis of a-si and a-sige solar cells including voltage-dependent photocurrent collection,” Progress in Photovoltaics: Research and Applications, vol. 5, pp. 151-168, 1997.

[10] William Shockley and Hans J. Queisser, "Detailed Balance Limit of Efficiency of p-n Junction Solar Cells", Journal of Applied Physics, Volume 32, pp. 510-519 (1961).

[11] Zulkefle, Ahmad Aizan, Maslan. Zainon, Zaiha. Sraf. Zakaria, Kamaruzzaman Sopian, And Nowshad Amin. "Numerical modeling of silicon/germanium (Si/Ge) superlattice solar cells." In Proc. of the 7-th WSeAS int. Conf. on Renewable energy sources, pp. 233-237. 2013.

[12] S.T.Chang, et.al., "Si/SiGe hetero-junction solar cell with optimization design and theoretical analysis", Thin Solid Films, vol. 519, pp. 5022-5025, 2011.

\section{Authors’ Profiles}

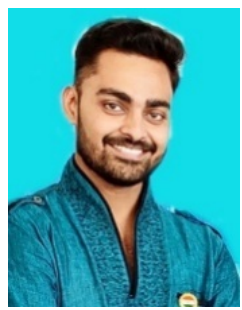

Ashish Kumar Singh is expecting his Master of Technology degree from National Institute of Technology Jamshedpur, India, in 2020. Presently, he is working as a system validation engineer in Intel, Bangalore. He has received shri mata vaishno devi shrine board gold medal from Prime Minister Narendra Modi for best undergraduate student in his B.Tech (Electronics \& Communications). He has contributed with his publication in several fields like photonics, silicon solar cells, irrigation system, power generation, machine learning and routing protocols.



Manish Kumar is presently working on Heterostructure Silicon and Germanium Alloy Based Thin Film Solar Cell Efficiency Analysis as his Master Thesis. He is expecting master's degree in communication system engineering from National Institute of Technology Jamshedpur. He has completed Bachelor of Technology in Electronics \& Communication engineering from Haldia Institute of Technology, West Bengal.



Dilip Kumar, currently an associate professor in National Institute of Technology Jamshedpur, Jharkhand, India. He is perusing his Ph.D in microwave engineering from National Institute of Technology Jamshedpur. He has 24 year of experience in teaching and also served in Indian Air Force as a flight engineer from 1987 to 1996. Presently he is carrying out research in Dielectric Investigation of Industrially Important material under Broadband Dielectric Spectroscopy. 
Professor S N Singh is Head of the Department of Electronics \& Communication Engineering in National Institute of Technology Jamshedpur, Jharkhand, India. He has worked on Development of PV Powered Intelligent Adaptive Power Inverter as Utility Interface for Use in AGRO - Based Applications as his research project. Published more than 30 International Conference and Journal papers. He is Member of Editorial Board of the Journals for IJECEE, IJEMR, IJECCT, IJETAE. He also has experience in R\&D NML (CSIR - A unit of Govt. of India) for 6 years.

How to cite this paper: Ashish Kumar Singh, Manish Kumar, Dilip Kumar, S N Singh. "Heterostructure Silicon and Germanium Alloy Based Thin Film Solar Cell Efficiency Analysis ", International Journal of Engineering and Manufacturing(IJEM), Vol.10, No.2, pp.29-40, 2020. DOI: 10.5815/ijem.2020.02.03 